\title{
PREVALÊNCIA DE SÍFILIS EM PARTURIENTES ATENDIDAS NA MATERNIDADE ESCOLA DE VALENÇA - RJ SEM A REALIZAÇÃO DE EXAMES PRÉ-NATAIS
}

\author{
Prevalence of syphilis in parturents seen at the maternity school of Valença -RJ \\ without having prenatal exams performed
}

\begin{abstract}
Débora Comin ${ }^{1}$
Raimundo Nonato Dias Junior ${ }^{1}$ Daniela Souto ${ }^{1}$

Elisabeth Valente Carvalho ${ }^{1}$ Lucas Vellasco de Mattos ${ }^{1}$ Jacqueline Travassos de Melo ${ }^{1}$
\end{abstract}

${ }^{1}$ Centro Universitário de Valença (UNIFAA) Valença (RJ)

\section{Autor correspondente:}

Lucas Vellasco de Mattos E-mail: lucas.mattos@faa.edu.br

\section{Como citar este artigo:}

COMIN, D.; DIAS JUNIOR, R.N.; CARVALHO, E.V.; MATTOS, L.V.; MELO, J.T. Prevalência de sífilis em parturientes atendidas na Maternidade Escola de Valença-RJ sem a realização de exames pré-natais. Revista Saber Digital, v. 14, n. 3, p. 4354, 2021

Data de Submissão: 13/11/21

Data de aprovação: 28/11/21 Data de publicação: 21/12/21

\section{(c) (1) (8)}

Esta obra está licenciada com uma licença http://creativecommons.org/licenses/by-nc/4.0/

\section{RESUMO}

Introdução: O perfil de infecções sexualmente transmissíveis (ISTs) continua crescendo em todo o mundo. Entre as principais consequências da infecção não tratada, destacam-se a transmissão vertical do Treponema pallidum e a associação com a infecção pelo vírus da imunodeficiência humana (HIV). Objetivo do estudo: Analisar a prevalência de sífilis e outras ISTs em parturientes atendidas na maternidade do hospital escola de Valença sem a realização de exames pré-natais. Material e métodos: Trata-se de um estudo retrospectivo com abordagem descritiva onde utilizou-se a análise de prontuários de gestantes atendidas na maternidade escola de Valença, RJ. Foi analisado o número de gestantes que realizaram os respectivos testes sorológicos para sífilis, HIV e Hepatite B. Resultados: Foram pesquisados 362 prontuários de gestantes, atendidas entre os meses de setembro de 2018 a julho de 2019. Observou-se que das 362 gestantes atendidas, 159 não realizaram as sorologias citadas acima. Tendo sido necessário, com isso, realizá-las no momento antecedente ao parto. Com relação à sífilis, em um total de 159 gestantes, obteve-se $11,32 \%$ de sorologias positivas. Constatou-se que a maioria das gestantes realizaram partos normais, em contrapartida, $16,3 \%$ destas foram submetidas a parto cesáreo. Conclusão: Através deste estudo foi possível observar que, uma parcela considerável de pacientes chega à maternidade sem os exames necessários para a realização de um parto com segurança. Há que se enfatizar a importância de um prénatal de qualidade, trazendo assim, um impacto positivo na redução da prevalência da sífilis congênita para o município de Valença.

Palavras-chave: Sífilis, HIV, Gestante.

\section{ABSTRACT}

Introduction: The profile of sexually transmitted infections (STIs) continues to grow around the world. Among the main consequences of untreated infection, vertical transmission of Treponema pallidum and an association with human immunodeficiency virus (HIV) infection stand out. Study objective: To analyze the prevalence of syphilis and other STIs in parturients seen at the maternity ward of the teaching hospital in Valença with no previous prenatal exams. Material and methods: This is a retrospective study with a descriptive approach which used the analysis of medical records of pregnant women seen at the school hospital maternity ward in Valença, RJ. The number of pregnant women who underwent the respective serological tests for syphilis, HIV and Hepatitis B was analyzed. Results: A total of 362 medical records of pregnant women attended between September 2018 and July 2019 were researched. It was observed that of the 362 pregnant women seen, 159 did not undergo the serologies mentioned above. As a result, it was necessary to perform them before giving birth. With regard to syphilis, in a total of 159 pregnant women, $11.32 \%$ of positive serology was obtained. It was found that most pregnant women had normal births. On the other hand; $16.3 \%$ of them underwent cesarean section. Conclusion: Through this study it was possible to observe that a considerable portion of patients arrives at the maternity ward without 
Prevalência de sífilis em parturientes atendidas na Maternidade Escola de Valença-RJ sem a realização de

exames pré-natais

Comin D, Dias Junior RN, Souto D, Carvalho EV, Mattos LV, Melo JT

the necessary exams for a safe delivery. The importance of quality prenatal care must be emphasized, thus bringing a positive impact on reducing the prevalence of congenital syphilis in the city of Valença.

Keywords: Syphilis, HIV, Pregnant.

\section{INTRODUÇÃO}

Entre as ISTs, a sífilis merece destaque, já que é uma doença infecciosa e sistêmica, de abrangência mundial e evolução crônica. É causada pela Espiroqueta Treponema pallidum, tem o homem como único hospedeiro, e reservatório. Sua transmissão pode ocorrer de forma sexual ou vertical, sendo mais frequente em grandes centros urbanos e afeta igualmente todas as camadas sociais (SOUZA, 2005).

A patogênese do treponema inicia-se pela penetração do agente por pequenas abrasões decorrentes da relação sexual. Logo após, o treponema atinge o sistema linfático regional e disseminação hematogênica. Após um período de incubação médio de 2 a 6 semanas, surge à lesão primária no local da inoculação, $O$ estágio secundário da doença está associado ao aparecimento de lesões cutâneo-mucosas e linfadenopatias generalizadas. (AVELLEIRA, 2006; MARADO, et al., 2013).

No Brasil, a sífilis continua, epidemiologicamente, como uma doença em ascensão. Desde 1986, foi incluída na lista de doenças de notificação compulsória na tentativa de facilitar e ampliar o diagnóstico, além de garantir o tratamento adequado, realizado de acordo com normas técnicas do Ministério da Saúde (MS) publicadas em 2004 e vigentes até o presente momento (HOLANDA, 2011).

Segundo o Ministério da Saúde (MS), a cada ano, 50 mil parturientes têm o diagnóstico de sífilis, com prevalência variando de 1,1 a 11,5\%, em função da assistência pré-natal e do grau de instrução materna. O resultado é que, ao ano, aproximadamente 12 mil nascidos vivos têm sífilis congênita no Brasil. Assim o controle da doença faz parte das metas do Pacto pela Saúde e a Organização Mundial de Saúde (OMS) projetava a eliminação da sífilis congênita até 2015. 
Mesmo com essa projeção, em várias regiões do Brasil identificam-se estudos que verificam as dificuldades no controle dessa infecção. (COSTA, 2013; LAFETÁ et al., 2016).

Segundo Brasil (2017), recomenda-se que o teste para a detecção da sífilis seja oferecido a todas as gestantes nos primeiros estágios da gravidez durante 0 atendimento pré-natal. Essa medida visa diagnosticar precocemente a doença na gestante, realizando o tratamento adequado e imediato, reduzindo assim as possibilidades do recém-nascido de adquirir a doença.

Os testes utilizados para o diagnóstico podem ser treponêmicos e não treponêmicos. O teste qualitativo indica a presença ou ausência de anticorpo na amostra. O teste quantitativo permite determinar o título de anticorpos. A titulação é importante para o diagnóstico e monitoramento da resposta ao tratamento. Isso porque a queda do título é indicação de sucesso do tratamento. O teste mais utilizado no campo sanitário é o VDRL (do ingles Venereal Disease Research Laboratory). Contudo, seu resultado positivo não confirma a infecção pelo T.pallidum e, portanto, não define o diagnóstico de sífilis. Após um resultado positivo realiza-se o teste treponêmico (FTA-ABS), específico para confirmar a infecção sifilítica (SÃO PAULO, 2016; BRASIL, 2017).

Entre as principais consequências da infecção não tratada, destacam-se a transmissão vertical do Treponema pallidum e a associação com a infecção pelo vírus da imunodeficiência humana (HIV). A coinfecção entre HIV e sífilis apresenta ação sinérgica, caracterizada tanto pela elevação da transmissibilidade do HIV quanto pela evolução atípica da infecção treponêmica (PINTO, 2014; LUPPI et al., 2018).

Ainda se associam à ocorrência de sífilis e coinfecção por HIV, uso de drogas, gravidez na adolescência, história de natimortalidade, comportamento sexual de risco, migração para grandes centros urbanos, acesso limitado aos cuidados de saúde e o não tratamento do parceiro infectado (MAGALHÃES et al., 2013). As ações para a prevenção e controle da sífilis, tais como, uso de preservativo, detecção precoce e tratamento adequado dos infectados e de seus parceiros sexuais, encontram-se disponíveis na rede básica de saúde e são de 
baixo custo. Ressalta-se que essas ações apresentam relação custo-benefício altamente vantajoso (LUPPI et al., 2018).

De acordo com o Ministério da saúde, deve ser realizado, em todas as gestantes, durante a primeira consulta pré-natal, o teste rápido diagnóstico antiHIV e/ou a sorologia anti-HIV, sendo repetidos no terceiro trimestre gestacional. Em caso de positividade, deve-se realizar o aconselhamento pós-teste e encaminhamento da gestante para o seguimento do pré-natal no serviço de atenção especializada em DST/AIDS de referência. Em relação à sífilis, o MS recomenda a realização de exames (teste rápido para a triagem da sífilis e/ou VDRL) logo na primeira consulta pré-natal (ou no primeiro trimestre), no terceiro trimestre e no parto. Diante dos casos positivos para sífilis em gestantes, o MS preconiza tratamento da gestante e do parceiro com penicilina benzatina, além da realização de exame mensal para controle de cura (ARAUJO, 2018).

Nesse contexto, ao refletirmos sobre as ISTs com ênfase na sífilis e suas complicações na gestação, nos reportamos à Maternidade do Hospital Escola de Valença e questionamos sobre a prevalência destas infecções nas parturientes, sem a realização de exames pré-natais, no momento da internação para realização do parto.

\section{MATERIAL E MÉTODOS}

Trata-se de um estudo retrospectivo com abordagem descritiva onde se utilizou a análise de prontuários de gestantes atendidas na Maternidade do Hospital Escola de Valença. Foram analisados o número de partos vaginais e cesáreos realizados bem como se as gestantes realizaram os respectivos testes sorológicos para sífilis, HIV e Hepatite B. Para a análise e visualização dos dados foi utilizado como ferramenta o programa Microsoft Excel. Este projeto foi submetido ao Comitê de Ética em Pesquisa com Seres Humanos do Centro Universitário de Valença UNIFAA e recebeu aprovação com CAAE: 10059219.8.0000.5246. 


\section{RESULTADOS E DISCUSSÃO}

Foram analisados 362 prontuários de gestantes em peri-parto, atendidas entre os meses de setembro de 2018 a julho de 2019. Os resultados relacionados ao tipo de parto realizado estão representados abaixo:

Gráfico 1 - Percentual de gestantes atendidas no Hospital Escola Maternidade de Valença conforme o tipo de parto.

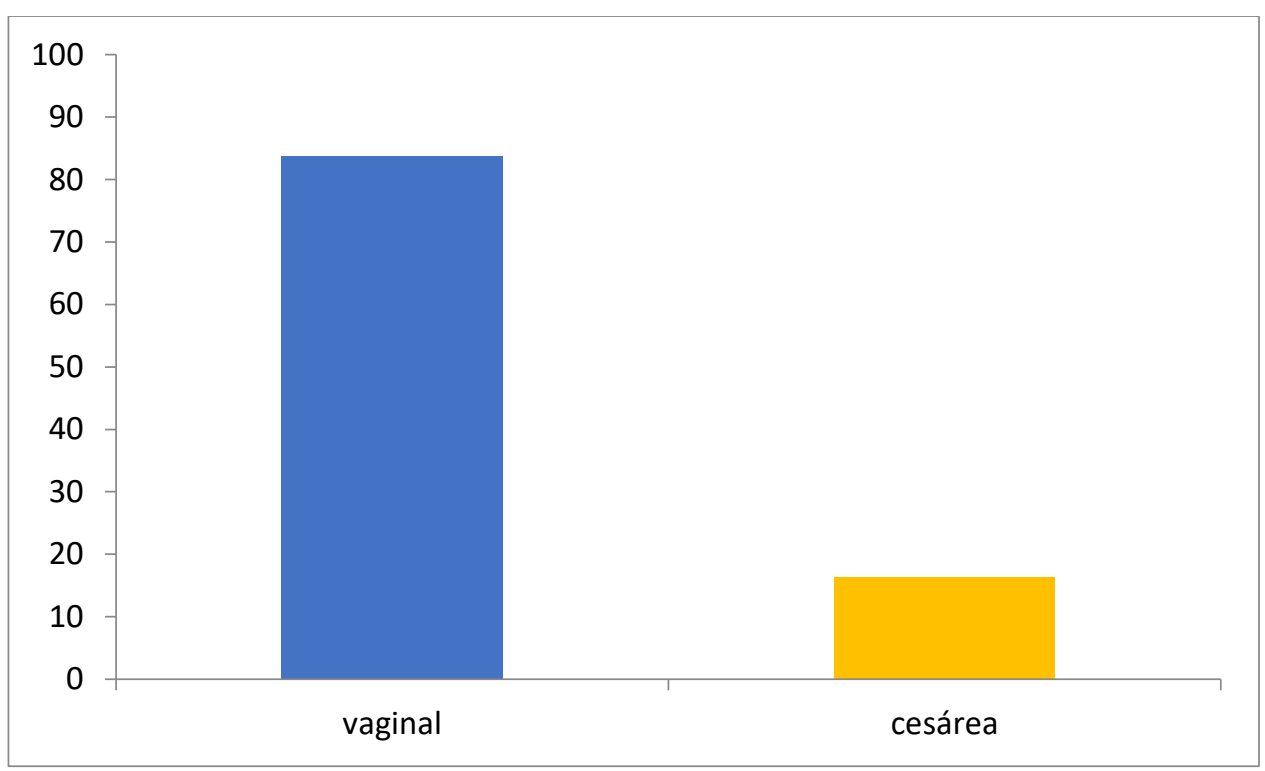

Fonte: Levantamento de dados obtidos dos prontuários do Hospital Maternidade Escola de Valença, 2019.

Conforme o gráfico acima das 362 gestantes atendidas na maternidade escola no decorrer de setembro de 2018 a julho de 2019 observou-se que a maioria dos partos foram vaginais $83,7 \%(n=303)$ em contrapartida $16,3 \%$ das gestantes $(n=59)$ foram submetidas a parto cesáreo. Estes resultados indicam a adoção de medidas direcionadas pelo ministério da saúde em que a realização do parto não deve traduzir medidas exacerbadas de intervenções médicas.

O avanço da obstetrícia contribuiu com a melhoria dos indicadores de morbidade e mortalidade materna e perinatais, no entanto permitiu o surgimento de um modelo que considera a gravidez, o parto e o nascimento como doenças 
e não como expressões de saúde. Desta forma expondo as mulheres e recémnascidos a altas taxas de intervenções, que deveriam ser utilizadas de forma parcimoniosa e apenas em situações de necessidade, e não como rotineiras (BRASIL, 2017). Não obstante, cabe ressaltar que, durante o parto normal aumentam as chances de transmissão vertical de ISTs tornando, neste caso, o parto cesário a opção mais segura e indicada para a prevenção de complicações obstétricas e neonatais (GIACOMINI, 2017).

Gráfico 2 - Comparação entre o total de gestantes atendidas e as que realizaram testes sorológicos no momento do parto para detecção de sífilis, HIV e Hepatite B.

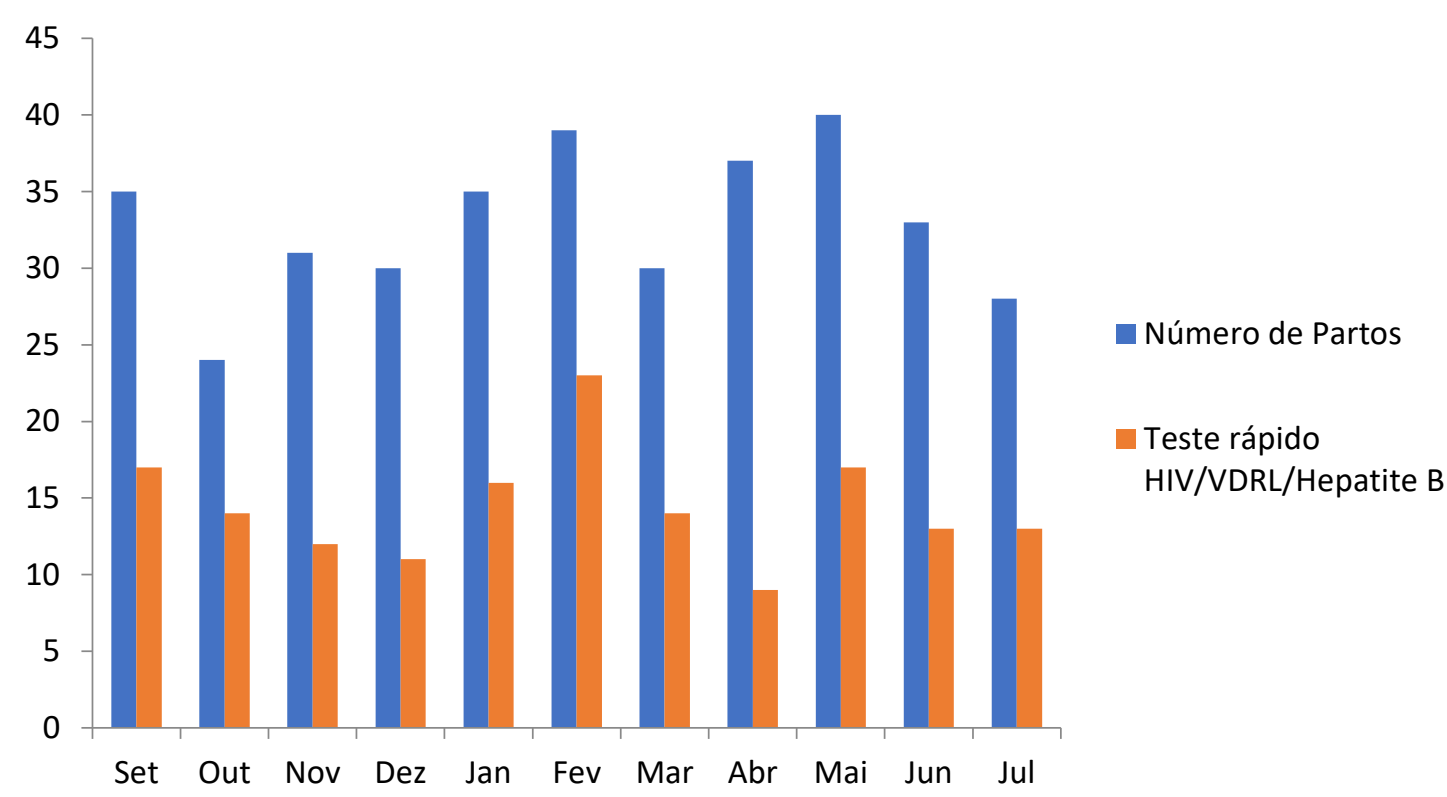

Fonte: Levantamento de dados obtidos dos prontuários do Hospital Maternidade Escola de Valença, 2019.

Das 362 gestantes atendidas, 159 fizeram rastreio para HIV, sífilis e hepatite B no momento antecedente ao parto. Estes dados indicam que 43,9\% das mulheres atendidas no momento do parto não tinham o rastreio adequado, como sugere o Ministério da Saúde, com consultas pré-natais adequadas e as sorologias de terceiro trimestre. Os resultados deste estudo indicam a importância da realização dos testes sorológicos na maternidade, uma vez que há gestantes que não realizam pré-natal e há aquelas que, mesmo tendo feito o 
Prevalência de sífilis em parturientes atendidas na Maternidade Escola de Valença-RJ sem a realização de

acompanhamento correto, não recebem o resultado e, por conseguinte, 0 tratamento durante a gravidez. Os resultados corroboram com uma pesquisa realizada com gestantes em 3 maternidades de Vitória, Espírito Santo. Tal estudo mostrou que cerca de $71(5,1 \%)$ das mulheres que não foram acompanhadas durante a gestação deixaram de ter acesso ao diagnóstico, não recebendo tratamento adequado $\mathrm{e}$, com isso, aumentando o risco de transmissão vertical (MIRANDA, et al., 2009). Diante do exposto faz-se necessário identificar as dificuldades de acesso dessas gestantes aos serviços de saúde.

O rastreamento das ISTs não se relaciona apenas a uma pessoa; ao contrário, estará sempre ligado a uma rede de transmissão. Quando não identificado e tratado o agravo no(s) parceiro(s), este se perpetua na comunidade e expõe o indivíduo à reinfecção, caso não se estabeleça a adesão ao uso de preservativos. Este rastreamento de ISTs é recomendado por subgrupo populacional. No caso de gestantes, recomenda-se que o rastreio para HIV e sífilis ocorra na primeira consulta do pré-natal (idealmente, no $1^{\circ}$ trimestre da gestação); no início do $3^{\circ}$ trimestre (28aㅗ semana); no momento do parto, em caso de aborto/natimorto, independentemente de exames anteriores. Para Hepatite B, deve ser realizado durante o primeiro trimestre. Outros micro-organismos, como Chlamydia trachomatis e Neisseria gonohrroeae também devem ser rastreadas na primeira consulta do pré-natal (BRASIL, 2019). 
Gráfico 3 - Percentual de gestantes com rastreio positivo para sífilis, HIV e Hepatite B na internação para o parto.

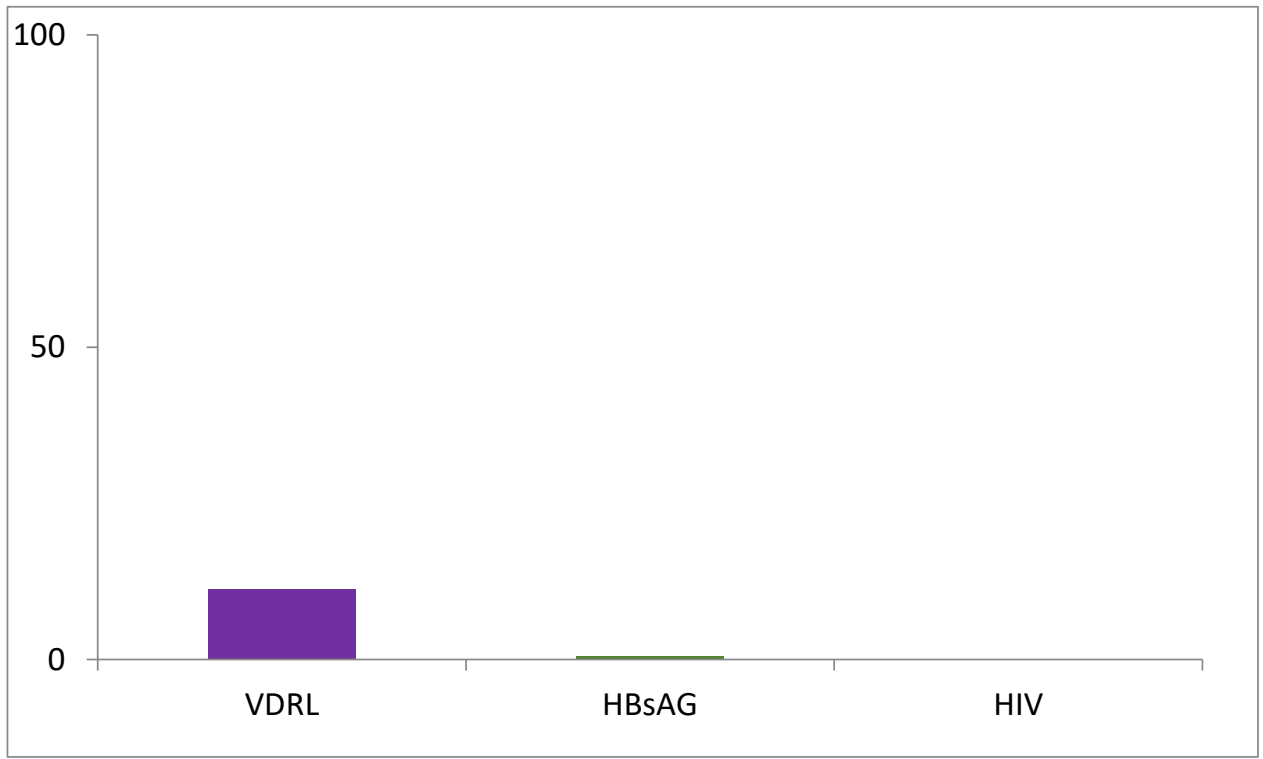

Fonte: Levantamento de dados obtidos dos prontuários do Hospital Maternidade Escola de Valença, 2019.

Com relação à sífilis, em um total de 159 gestantes obteve-se 11,32\% $(n=18)$ de sorologia positiva na internação antecedente ao parto. Quanto ao rastreio para HIV, todas as pacientes investigadas no momento da internação tiveram sorologia negativa. Outra IST investigada foi a hepatite $B$, onde foi identificado apenas 1 (uma) gestante positiva para hepatite $B$, ou seja, menos de $1 \%$ das pacientes avaliadas. Os resultados apresentados não evidenciaram uma correlação da coinfecção sífilis/ HIV, diferentemente do que é mostrado em outros estudos.

Segundo Brasil (2019) a prevalência de sífilis é maior entre as pessoas vivendo com HIV que entre as pessoas negativas para este vírus. Este estudo sugere que a alta taxa de sífilis é mais provavelmente causada por fatores comportamentais do que por fatores imunológicos. A aquisição de sífilis e outras ISTs em portadores do HIV confirmam a vulnerabilidade e a falha na adesão às orientações de prevenção. Pessoas com comportamento sexual de alto risco muitas vezes têm acesso limitado aos cuidados de saúde, devido a questões econômicas e/ou estigmatização social. 
Acosta (2016), mostrou em seu estudo, que de 1500 gestantes positivas para HIV com partos no período de 2010 a 2013, 155 (10,3\%) também estavam infectadas por sífilis, correspondendo a uma taxa de coinfecção HIV/sífilis de 10,2\%. Conclui-se que a prevalência de HIV e sífilis em parturientes é elevada e está na dependência da qualidade da assistência pré-natal.

Lafetá et al., (2016) descreve na assistência pré-natal, que 43\% das gestantes com diagnóstico de sífilis realizaram pelo menos 6 consultas, na qual aproximadamente metade dos casos (48,4\%) conseguiu iniciar 0 acompanhamento ainda no $1^{\circ}$ trimestre de gestação. Contudo, a maioria das gestantes apresentou diagnóstico tardio no momento do parto ou da curetagem $(62,4 \%)$ e, ainda, segundo os autores, nenhum caso foi considerado adequadamente tratado segundo as recomendações do Ministério da Saúde. Segundo os autores, 33,3\% não receberam nenhum tratamento, e a totalidade foi considerada inadequadamente tratada, principalmente devido ao não tratamento do parceiro. O estudo também mostrou que houve um aumento gradual no número de casos de sífilis tanto entre gestantes quanto entre recémnascidos ao longo dos anos analisados.

Diante do exposto, faz-se necessário ressaltar a importância do pré-natal quanto ao diagnóstico e tratamento das ISTs, bem como aprimorar as medidas que levem à população informações sobre transmissão e prevenção com vistas a redução de novos casos.

\section{CONCLUSÃO}

Através deste estudo foi possível observar que, mesmo com o trabalho da estratégia de saúde da família, disseminada em vários bairros do Município de Valença, e tendo como foco uma assistência de qualidade para as gestantes, uma parcela considerável das gestantes chegam à maternidade para o trabalho de parto sem as sorologias necessárias para a realização de um parto com segurança. 
Espera-se que este trabalho colabore com o raciocínio dos profissionais da saúde, para uma visão holística da gestante, principalmente no peri-parto, onde além da sífilis, IST em ascensão em nosso país, outras infecções que podem trazer risco à saúde do $\mathrm{RN}$, sejam devidamente investigadas e tratadas. É de suma importância que, durante o pré-natal, os profissionais de saúde estejam atentos as sorologias e testes de triagem de acordo com as políticas públicas do ministério da saúde para a realização do parto. Caso contrário, o rastreio na maternidade torna-se fundamental para garantir o bem-estar, da puérpera e do RN. Para a erradicação da sífilis congênita e outras infecções de transmissão vertical, faz-se necessário priorizar o diagnóstico precoce e assegurar o correto tratamento da gestante. Por fim, enfatiza-se a importância de um pré-natal de qualidade, trazendo assim, um impacto positivo na redução da prevalência da sífilis congênita para o município de Valença.

\section{REFERÊNCIAS}

ACOSTA, L.M.W.; GONÇALVES, T.R.; BARCELLOS, N.T. Coinfecção HIV/sífilis na gestação e transmissão vertical do HIV: um estudo a partir de dados da vigilância epidemiológica. Rev Panam Salud Publica, v. 40, n. 6, p. 435-42, 2016.

ARAUJO, E.C.; MONTE, P.C.B.; HABER, A.N.C.A. Avaliação do pré-natal quanto à detecção de sífilis e HIV em gestantes atendidas em uma área rural do estado do Pará, Brasil. Rev Pan-Amaz Saude, Ananindeua, v. 9, n. 1, p. 3339, mar. 2018.

AVELLEIRA, J.C.R.; BOTTINO, G. Sífilis: diagnóstico, tratamento e controle. An. Bras. Dermatol., Rio de Janeiro, v. 81, n. 2, p. 111-126, 2006.

BRASIL. Ministério da Saúde. Secretaria de Ciência, Tecnologia e Insumos Estratégicos. Departamento de Gestão e Incorporação de Tecnologias em Saúde.

Diretrizes nacionais de assistência ao parto normal: versão resumida [recurso eletrônico] / Ministério da Saúde, Secretaria de Ciência, Tecnologia e Insumos Estratégicos, Departamento de Gestão e Incorporação de Tecnologias em Saúde. - Brasília: Ministério da Saúde, 2017. 
BRASIL. Ministério da Saúde. Secretaria de Vigilância em Saúde. CoordenaçãoGeral de Desenvolvimento da Epidemiologia em serviços. Guia de Vigilância em Saúde: volume único. Mistério da saúde, Secretaria de Vigilância em Saúde, Coordenação-Geral de Desenvolvimento da Epidemiologia em Serviços - $2^{\underline{a}}$ ed - Brasília: Ministério da Saúde, 2017.

BRASIL. Ministério da Saúde. Secretaria de Vigilância em Saúde. Departamento de Doenças de Condições Crônicas e Infecções Sexualmente Transmissíveis. Protocolo Clínico e Diretrizes Terapêuticas para Atenção Integral às Pessoas com Infecções Sexualmente Transmissíveis (IST)/Ministério da Saúde, Secretaria de Vigilância em Saúde, Departamento de Doenças de Condições Crônicas e Infecções Sexualmente Transmissíveis. - Brasília: Ministério da Saúde, 2019.

COSTA, C.C. et al. Sífilis congênita no Ceará: análise epidemiológica de uma década. Rev. esc. enferm. USP, São Paulo, v. 47, n. 1, p. 152-159, 2013.

GIACOMINI, M.R.; SOUZA M. Transmissão vertical de infecções sexualmente transmissíveis: uma revisão narrativa. Ciências da Saúde, Santa Maria, v. 18, n. 2, p. 409-417, 2017.

HOLANDA, M.T.C.G. et al. Epidemiological pattern of inherited syphilis in the City of Natal, State of Rio Grande do Norte, Brazil, from 2004 to 2007.Epidemiol. Serv. Saúde, Brasília, v. 20, n. 2, p. 203-212, 2011.

LAFETÁ, K.R.G. et al. Sífilis materna e congênita, subnotificação e difícil controle. Rev. bras. epidemiol. São Paulo, v. 19, n. 1, p. 63-74, 2016.

LUPPI, C.G. et al. Fatores associados à coinfecção por HIV em casos de sífilis adquirida notificados em um Centro de Referência de Doenças Sexualmente Transmissíveis e Aids no município de São Paulo, 2014. Epidemiol. Serv. Saúde, Brasília, v. 27, n. 1, e20171678, 2018.

MAGALHAES, D.M.S. et al. Sífilis materna e congênita: ainda um desafio. Cad. Saúde Pública, Rio de Janeiro, v. 29, n. 6, p. 1109-1120, 2013.

MARADO, D. et al. Sífilis: uma causa rara de hepatite colestática. J Port Gastrenterol., Lisboa, v. 20, n. 2, p. 70-73, 2013.

PINTO, V.M. et al. Prevalência de Sífilis e fatores associados a população em situação de rua de São Paulo, Brasil, com utilização de Teste Rápido. Rev. bras. epidemiol., São Paulo, v. 17, n. 2, p. 341-354, 2014.

MIRANDA, A.E. et al. Prevalência de sífilis e HIV utilizando testes rápidos em parturientes atendidas nas maternidades públicas de Vitória, Estado do Espírito 
Prevalência de sífilis em parturientes atendidas na Maternidade Escola de Valença-RJ sem a realização de exames pré-natais Comin D, Dias Junior RN, Souto D, Carvalho EV, Mattos LV, Melo JT

Santo. Revista da Sociedade Brasileira de Medicina Tropical, São Paulo, V. 42, n. 4, p. 386-391, 2009.

SÃO PAULO. Secretaria de Estado da Saúde. Centro de controle de Doenças. Programa Estadual de DST/Aids. Centro de Referência e Treinamento DST/Aids. Guia de bolso para o manejo da sífilis em gestantes e sífilis congênita. São Paulo: Secretaria de Estado da Saúde. 2016. 112p.

SOUZA, E.M. Há 100 anos, a descoberta do Treponema pallidum. An. Bras. Dermatol., Rio de Janeiro, v. 80, n. 5, p. 547-548, 2005. 